\title{
Coulisses
}

Revue de théâtre

\section{Retour sur le colloque théâtre et sciences des 14, 15, 16 mai 1998 à Besançon}

En partenariat avec le Centre Jacques-Petit, le laboratoire Littérature et Histoire des pays de langues européennes, l'École doctorale LangagesEspace-Temps, le Théâtre de l'Espace, scène nationale de Besançon, le service des activités culturelles du CROUS

\section{Lucile Garbagnati}

\section{(2) OpenEdition}

\section{Journals}

Édition électronique

URL : https://journals.openedition.org/coulisses/5680

DOI : $10.4000 /$ coulisses. 5680

ISSN : 2546-9460

\section{Éditeur}

Presses universitaires de Franche-Comté

\section{Édition imprimée}

Date de publication : 1 janvier 1999

Pagination : $54-55$

ISBN : 2-913322-09-3

ISSN : $1150-594 X$

\section{Référence électronique}

Lucile Garbagnati, «Retour sur le colloque théâtre et sciences des 14, 15, 16 mai 1998 à Besançon », Coulisses [En ligne], 19 | Hiver 1999, mis en ligne le 18 octobre 2019, consulté le 12 janvier 2022. URL http://journals.openedition.org/coulisses/5680 ; DOI : https://doi.org/10.4000/coulisses.5680

Ce document a été généré automatiquement le 11 janvier 2022. 


\section{Retour sur le colloque théâtre et sciences des 14, 15, 16 mai 1998 à Besançon}

En partenariat avec le Centre Jacques-Petit, le laboratoire Littérature et Histoire des pays de langues européennes, l'École doctorale LangagesEspace-Temps, le Théâtre de l'Espace, scène nationale de Besançon, le service des activités culturelles du CROUS

\section{Lucile Garbagnati}

1 L'intérêt de ce colloque théâtre et sciences et son originalité, confirmés maintenant par la réussite de la manifestation, reposent sur un triple constat :

1. Nous vivons dans un monde scientifique et le théâtre en parle peu ou prou.

2. Pour parler de théâtre, il faut en montrer.

3. Le thème étant à la fois d'une actualité constante et peu médiatisée, il fallait un support à la fois attractif et pédagogique : le théâtre de rue et l'action culturelle.

\section{Une triple organisation}

- 27 communications scientifiques sur les 29 prévues. Les textes sont d'ailleurs publiés dans les Actes ${ }^{1}$.

- 6 spectacles de genres différents pour toucher des publics divers avec des partenaires institutionnels spécifiques, dans des lieux variés.

- Action culturelle auprès des classes d'école primaire.

2 Les communications de toutes disciplines (biologie, physique, sociologie, psychopédagogie, littérature française et étrangère, théâtrologie) ont pris tantôt la forme d'exposés scientifiques tantôt celle de comptes rendus d'expériences (Muséum d'histoire naturelle, P.A.E., École d'ingénieurs...). Elles ont été suivies par une vingtaine d'auditeurs régulièrement inscrits au colloque, en particulier des responsables de troupes théâtrales bisontines, sans parler d'un auditoire plus irrégulier. 
3 Les spectacles ont élargi considérablement l'audience du colloque que ce soit dans la salle du restaurant universitaire, dans la rue, au Théâtre de l'Espace. Ils ont à la fois alimenté la réflexion et servi de caisse de résonance à la problématique.

\section{Un partenariat transdisciplinaire : la participation des centres de recherche universitaire}

4 Le colloque a été impulsé et organisé par une structure théâtrale étudiante et amateur : le Théâtre Universitaire de Franche-Comté soutenu par le Centre de recherches Jacques-Petit, le Laboratoire de Littérature et Histoire des pays de langues européenne, l'École Doctorale Langages-Espaces-Temps. Ils ont apporté, avec leur concours financier, la garantie de la valeur scientifique du colloque. Soulignons qu'il a obtenu des fonds du B.Q.R. de l'Université. Il est donc reconnu comme s'inscrivant dans les lignes de la recherche universitaire bisontine.

\section{Le partenariat avec les structures culturelles et théâtrales : la représentation des spectacles}

5 Le choix des spectacles a été fondé sur le souci de montrer la variété potentielle du spectacle scientifique, ce qui implique, en gardant en tête le public à toucher, le choix des spectacles, le lieu de la représentation, donc le partenariat. Dans cette perspective, le partenariat avec le service des activités culturelles du CROUS et avec le Théâtre de l'Espace s'est montré des plus efficaces.

6 Sans entrer dans le débat sur le rapport entre conscientisation et théâtre, nous voulions toucher le public le plus vaste possible. C'est pourquoi nous avons pensé qu'il fallait à la fois des "petites formes" dans le cadre des salles de conférence, des formes ouvertes à tous : le théâtre de rue, et, évidemment, le public des salles de théâtre ; dans ces dernières, des spectacles pour enfants et pour adultes.

\subsection{Le partenariat avec le CROUS}

7 Dans son souci d'animation du Campus, le service des activités culturelles du CROUS a pris en charge le spectacle de rue Tout va mal de la compagnie Embarquez. Soulignons que, depuis, il a reçu différents spectacles de cette troupe qui s'est produite sur l'esplanade du restaurant universitaire de La Bouloie.

\subsection{Le partenariat avec la scène nationale de Besançon}

8 Celui-ci s'est avéré capital pour la réussite de la manifestation tant pour la sélection et l'accueil que pour la diffusion des spectacles professionnels non régionaux. 


\section{En fonction du public et du lieu, trois types de spectacles ont été représentés :}

- 2 spectacles de petite forme, sans aucune exigence technique :

- Mickey l'ange par la compagnie du $8^{\text {ème }}$ ciel (Lille) dans le cadre même du colloque,

- Une amitié scientifique et littéraire : Léon Burp et Marcel Gotlib de Georges Pérec par le Théâtre Universitaire de Franche-Comté au cours du buffet.

- 2 spectacles de rue :

- l'un pour toucher le tout public: Container follies, de et par Manches à Balais Korporation, Place Cassin,

- l'autre pour toucher le public étudiant: Tout va mal par la compagnie Embarquez, devant le restaurant universitaire de La Bouloie.

- 2 spectacles en salle impliquant donc le Partenariat avec une structure professionnelle, le Théâtre de l'Espace, scène nationale de Besançon :

- un spectacle pour enfants, Zéphyrin et le météore d'or, par la compagnie Les bateleurs de la science,

- un spectacle pour public de théâtre : L'homme de plein vent de Pierre Meunier.

Comme espéré, chaque spectacle a été suivi par un public particulier donnant au colloque une atmosphère spécifique influant sur les rapports du groupe.

Parterre d'une soixantaine d'enfants et de mamans et un nombre de passants difficile à évaluer pour Container follies. Beaucoup de curiosité sympathique et de questions sur les poubelles. Autant d'étudiants pour Tout va mal. Rires et questions aux comédiens, sur leur travail plus que sur la médecine. Le buffet du colloque rassemblait les participants plus une trentaine d'invités qui se sont pris au jeu de la parodie de la communication universitaire de Pérec. En gros, les formes moins conventionnelles de spectacle ont attiré de façon suivie environ 150 à 200 personnes, sans parler des passants et des arrivées de la dernière heure.

Ceux de l'Espace Planoise :

- L'homme de plein vent : 220 spectateurs.

-Zéphyrin et le météore d'or, 414 spectateurs, par groupes d'environ 60 enfants, avec des enfants des classes des écoles primaires de Champagne, de Bourgogne, du Rosemont, du Centre d'Éducation Sainte-Odile, des Francas, de l'A.R.E.T.E.

\section{Conclusions non conclusives}

Il ressort de ces deux journées et demi de réflexion et de confrontation quelquefois vives entre théoriciens, praticiens, responsables de structures, qu'un vaste chantier est ouvert ; il se situe d'ailleurs dans une perspective historique qu'il s'agit de redécouvrir.

Sur l'organisation générale du colloque : chacun s'est félicité de sa richesse, du nombre et de la qualité des communications et des spectacles représentés. Toutefois un regret a été émis sur le manque de temps laissé pour la critique des spectacles.

De l'avis général une pareille manifestation devrait se reproduire et Besançon pourrait devenir un lieu d'échanges privilégié autour de cette problématique : théâtre et sciences. 
Des propositions ont été émises :

- Constituer une banque d'informations sur le répertoire et les manifestations par internet et sur support papier. Dans un premier temps Coulisses, la revue du Théâtre Universitaire de Franche-Comté, peut servir de relais.

- Organiser un colloque régulier, tous les deux ans, pour mesurer les acquis et évaluer les manques.

- Organiser un festival de théâtre sur le thème théâtre et sciences.

\section{NOTES}

1. Voir le compte rendu de Christian Pratoussy, page suivante. 